\title{
Sonification of Planetary Orbits in Asteroid Belts
}

\author{
Michael Quinton ${ }^{\dagger}$ \\ School of Computing \\ Edinburgh Napier University \\ Edinburgh \\ michael.a.p.quinton@gmail.com
}

\author{
Iain McGregor \\ School of Computing \\ Edinburgh Napier University \\ Edinburgh \\ i.mcgregor@napier.ac.uk
}

\author{
David Benyon \\ School of Computing \\ Edinburgh Napier University \\ Edinburgh
}

\begin{abstract}
This study investigates the design and evaluation of a sonification designed to detect any planets orbiting within an asteroid belt of an exosolar system. The interface was designed for an astronomer who studies this phenomenon. User centered design methods were applied to create an accurate sonification of the data that could allow the astronomer to perceive possible planetary movements within an asteroid belt. The sonification was developed over three stages: A requirements gathering exercise inquiring about the data that the astronomer uses in her work. A design and development stage based on the findings of the requirements gathering and the third stage, an evaluation of the sonification design. The sonification effectively allowed the astronomer to immediately detect a planet orbiting within an asteroid belt. Multiple parameter mappings provide richer auditory stimuli that are more semantical to the user. The use of more familiar, natural sounding sound design led to a clearer comprehension of the dataset. The use of spatial mapping and movement allowed for immediate identification and understanding of the planet's course through the asteroid belt.
\end{abstract}

\section{CCS CONCEPTS}

Human-centered computing $\rightarrow$ Interaction design $\rightarrow$ Interaction design process and methods $\rightarrow$ User centered design.

\section{KEYWORDS}

Sonification, Parameter mapping sonification, Exoplanet hunting, Astronomy, User centered design, Grounded theory

\section{ACM Reference format:}

Michael Quinton, Iain McGregor and David Benyon. 2021. Sonification of planetary orbits in asteroid belts. In Proceedings of Audio Mostly (AM'21). ACM, Trento, Italy, 8 pages. https://doi.org/10.1145/0000000.0000000

\section{Introduction}

This study explored whether sonification can be successfully applied to a dataset representing the movement of a planet in an asteroid belt. The movement is reflected through the collision of asteroids that occur in the planet's trajectory as it orbits around its parent star in an Exosolar system. Neither the orbit nor the nature of the planet varies over time, but its gravitational force has an effect on the surrounding asteroid belt. Asteroids are pushed out of the way which causes a ripple effect (wave) throughout the belt. The angle of the belt is determined by the axial tilt of the central star around which it rotates. The data is a simulation of an Exosolar system designated as Beta $(\beta)$ Pictoris.

Permission to make digital or hard copies of part or all of this work for personal or classroom use is granted without fee provided that copies are not made or distributed for profit or commercial advantage and that copies bear this notice and the full citation on the first page. Copyrights for third-party components of this work must be honored. For all other uses, contact the owner/author(s).

AM'21, September 1-3, 2021, Trento, Italy

(C) 2021 Copyright held by the owner/author(s). 978-1-4503-0000-0/18/06 ...\$15.00

https://doi.org/10.1145/0000000.0000000
The asteroid collisions indicate the meeting point of two bodies where an impact occurs, reflecting a degree of complexity of spatial movement and localization. Chowning [1], states that when one listens to a sound one also expects to understand where the source is coming from and its context within the given space. This suggests that spatial mapping is more likely to provide familiarity to the listener and a more accurate representation of a natural occurrence.

Sonification systems tend to be less effective when complex data is represented through linear audio [2]. Spatiality provides depth to a flat, sonic representation [3]. Sound is spatial by nature, propagating and interacting with a given environment providing directionality and source location [4]. Surround sound extends beyond this, giving more contextual and directional definition by transmitting more auditory data [5]. Sound is explicitly related to the perception of time and can evoke meaning within a listener [6].

To convey these complex concepts clearly to an astronomer usercentered and task-based design methods could help create a successful sonification design $[7,8]$. These methods can help a designer to attain users' concepts in relation to data and understand the meaning and social significance an object or event may convey [9]. Various sonification designs have used familiarity in their work $[10,11,12]$. Sounds can work as a narrative to convey recognisable concepts and quantities related to objects. Sometimes a sound does not represent an object directly but articulates a related concept or event. In film a principle known as idea-associative comparison montage is where sounds are chosen that are congruous to an event on screen. Apparently disassociated, yet conceptually related events are used to strengthen a basic notion [13].

In this study planet detection was based upon colliding asteroids being affected by a planet's trajectory. Various concepts arise concerning the nature of the collisions. How an asteroid would sound when striking another and how this would reflect the existence of a much bigger planet causing these collisions. Bødker and Klokmose [14] describe how designers who aim to produce more natural and intuitive user interfaces apply Fauconnier and Turner's theory of Conceptual Blends [15]. This theory concerns creating a qualified match between two mental spaces, and selective components from these two inputs are blended together into a third mental space. The mental space is described as minute partial concepts formed from thought and dialogue for purposes of understanding and action. MacDonald and Stockman [16] used this approach to create an auditory display design theory where User Task design methods were blended with film compositions to draw parallels between different compositional processes. Requirements gathering was compared to Scene spotting, Conceptual design to Compositional arrangement, Detailed design to Musical scoring. 
Sonification designs sometimes fall-short if they fail to account for the vagaries of human perception and cognition [17]. The evolution in the technology of data representation is widening perspectives and possibilities, similar evolutions in sonifications can also manifest and open new vistas for scientists. Csikszentmihalyi and Halton [18] argue that there is an intimate, symbiotic relationship between humans and the tools they create and use, which also reflect the nature of a sense of self. User centered design methods can allow a designer to reach this deeper level of understanding the tool as an extension of the user and reflect this within the design. This level of intimacy was recognised by Laurel [19] who put forward the hypothesis that when people use computers, they tap into representational worlds similar to theatre and the interaction between the audience and the stage. In the more evolved practices of sound design for theatre, film, and video games, designers have devised empirical methods to convey meaning through the narrational qualities of sound [20].

Sonification design points towards investigating listening trends for a deeper understanding of user interface interaction. Listening gives active attention to extract information from a sonic environment [21]. Oliveros [22] suggests an expanded level of learning where sound carries intelligence, ideas, feelings and memories. Ceraso [23] states listening should go beyond ear perception, but also felt physically by the body.

Chion [24] described three modes of listening. Casual is the most common form where it is intended to gather information about the cause or source. This applies to seen and unseen sources, where in the case of the latter, the origin is identified by knowledge or prognosis. Semantic is a communicable cipher or lingual form of interpretation. This mode probes for meaning. Reduced focuses on the attributes of a sound regardless of cause or meaning. The sound is an observable article and listening is focused on physical properties of the object free from precognitive interpretations or meaning. Tuuri et al. [25] developed five more modes of listening to Chion's [24] three. Reflexive is an instinctive reaction to a sound that immediately captures attention. Connotative are the immediate associations gathered pre-consciously. Empathetic focuses on emotional cues. Functional analyses the purpose of a sound in relation to a specific function. Critical is analytical and is used for judging of aesthetics and interpretation.

It is believed that sonification could help an astronomer detect a planet in an asteroid belt situated in an Exosolar system. Data suggests that a planet causes asteroids to collide as it passes through a field of debris and makes the belt warp into a wave reflecting a planet's existence. User centered design methods will be used and a sonification design built upon the data. The results present the possible sonic mappings to fit the dataset and help determine the aesthetics of the model. There will be an iterative design process, the final version will be evaluated, and results presented.

\section{Requirements Gathering}

A semi-structured interview was conducted with an astronomer (AB), a female in her early thirties who had been working as a professional astrophysicist for approximately 8 years. Her work involved the development of a 3D visual simulation called SMACK (Super particle Model/Algorithm for Collision in Kuiper belts) which is a model of an asteroid belt situated in an exosolar system. $\mathrm{AB}$ considers her hearing and eyesight for her age to be normal and has had musical training but no experience in professional audio. She is familiar with the concept of sonification and wanted to use it to see if it would speed up the process of detecting a planet within the asteroid belt, and to grasp a deeper understanding of the planetary movement and related asteroid collisions.

The interview consisted of 22 main questions and additional supplementary questions, aimed to cover three aspects: The nature of the dataset, identifying parameter mapping of the data and gathering information about the sonification design. The interview was conducted on-line and lasted roughly about one hour. Being qualitative, it did not require a pilot study, allowing questions to form correspondingly to the responses. The recording of the interview was transcribed and coded.

Grounded Theory was used to gather data in order to guarantee the flexibility needed to develop the sonification prototype with minimal partiality [26]. This method can be coded using a three-pass system (initial, intermediate and advanced). The initial stage reduces the data into smaller components which are then compared to similar data from the same or other sources, and questions formed regarding the relevance of the findings. Intermediate coding is used to develop categories, and relationships are identified. Advanced coding forms the theory as a narrative for the researcher and any gaps in the theory can be recognised and addressed [27].

The gravitational perturbations of planets can create observable morphological characteristics in asteroid belts. Planets are detected by observing debris discs and collisions of particles across a disc's plane. As the planet moves through the center of the disc system it creates a spiral of planetesimals (asteroids) that collide with each other. The spiral is a result of the planet's elliptical orbit. The disc is also warped due to the planets orbital tilt [28]. The data, which is a simulation, consists of four columns (see Figure 1). The first represents time measured over a million days. The second column represents the horizontal plane, or the $\mathrm{X}$ axis measured in astronomical units (AU). The third column represents the vertical plane, or $\mathrm{Y}$ axis measured in astronomical units (AU) and the fourth column details the asteroids' collision data.
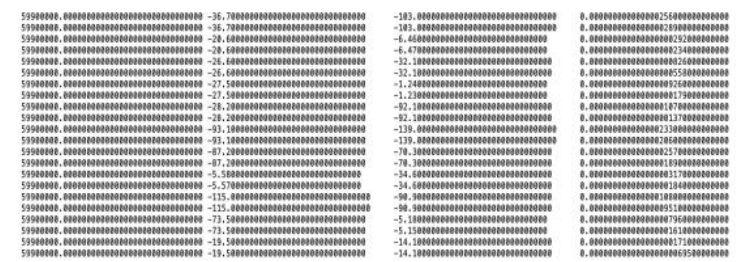

Figure 1: Example of the dataset

Pitch was one of the main parameter mappings where lower pitch represented larger asteroids and higher pitch smaller ones. The other main mapping was spatial attributed to the $\mathrm{X}$ axis which indicated spatial position of collisions occurring across a disc's plane. This was distributed on a Surround sound configuration with the listener in a heliocentric position with the disc orbiting around them. There was also the choice to select Stereo where a listener would face the disc on a horizontal plane to hear collisions occurring from left to right. This option would allow the 
sonification to be used with headphones. It was decided with $\mathrm{AB}$ that the sonification should sound like the familiar sound of ice breaking to assist discernment. Other parameter mappings such as timbre and amplitude indicated intensity of collisions. Larger objects would have less clear timbre but be louder due to their increased mass and smaller objects clearer in timbral qualities but lower in amplitude.

The data was provided in text format. $\mathrm{AB}$ would have control over playback speed for slowing down or speeding up. She could choose specific points of interest in the dataset for comprehensive listening. The playback needed a loop function for repeated listening of a data segment. $\mathrm{AB}$ also requested a recording function so that it could be played back at any time and on any supporting device platform. This function would also be convenient for playback of the sonification at conferences and other public engagement activities.

\section{Sonification Design}

The requirements gathering provided the basis for the sonification design and $\mathrm{AB}$ was involved in the iterative sound design process. Figure 2 displays the codes formulated using Grounded Theory.

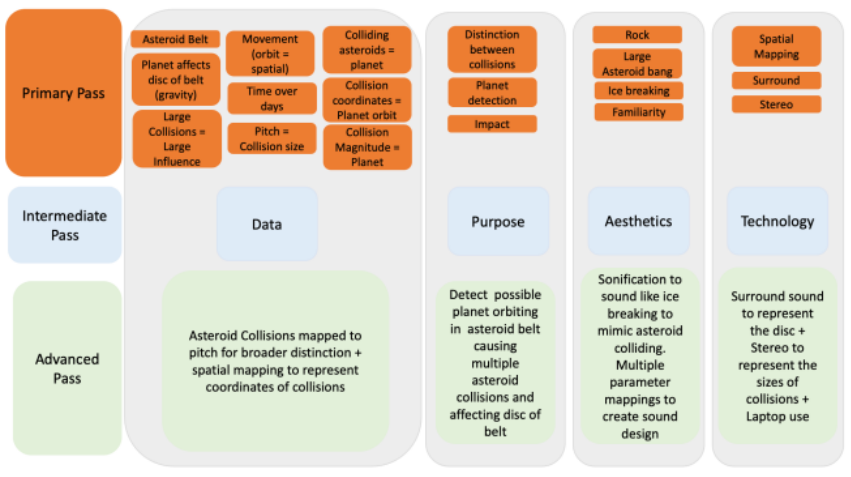

Figure 2: Sonification design criteria

The sonification was developed using subtractive synthesis engines. The first synth added pitch to a clap sound. Two oscillators added harmonics to the signal. Both were sawtooth waves and oscillator two was slightly detuned by 0.4 in a MIDI scale ranging from 0 to 1 giving the clap richer tonal properties. Sawtooth waves contain odd and even harmonic generating clear and harsh sounds [29]. The use of two waves adds distortion to a signal making it sharper and dissonant, closer to the sound of breaking ice and is more impacting and explosive. The second synth created the sound of a drum machine clap to give the explosive impact of an asteroid collision and mimic the sound of breaking ice which would sound more like a clap. To have a clean, sharp signal it was thought that subtractive synthesis would be the most effective. This technique allows a spectrally rich signal to be filtered to eliminate unwanted artefacts [30]. Changes in filter settings would determine the sharpness of smaller asteroids and the more booming larger ones.

Pitch (collision data) varied between $60 \mathrm{~Hz}$ to $4 \mathrm{kHz}$ allowing consistency in reproduction between datasets. Low pitch represented larger asteroid collisions and high pitch smaller ones. The lower frequency was set to $60 \mathrm{~Hz}$ to allow enough range for low frequency explosions to manifest. In the higher range, the limit was capped at $4 \mathrm{kHz}$. Any frequencies propagating beyond that point would only result from added harmonics enhancing the timbre rather than contributing to the pitch. These frequencies would still be within a listener's audible range. Short durational tones produce energy at frequencies other than the nominal frequency. The shorter the pulse the broader the bandwidth [31]. Short duration pitch distinction would be most accurate up until a $5 \mathrm{kHz}$ cap [32].

Other mappings were used to help create the ice-breaking sound and distinction in various sizes of the colliding asteroid impacts. A familiar sounding sonification could strengthen associative conceptualization related to the material a sound represents [33]. Breaking ice is an environmental sound and are usually processed in the brain similarly to speech [34]. The intention is to create a clear and immediate understanding of the parameter mappings.

In the first synthesizer both Oscillators were set to full volume. Cutoff varied with frequency. Higher pitched signal had higher Cutoff settings which gave smaller collisions more clarity. Table 1 shows the ranges of the changes in these variables in accordance with the collision data and unchanging fixed synth parameters.

Table 1: Sonification design Subtractive Synthesizer 1

\begin{tabular}{|l|l|l|l|}
\hline Parameters & $\begin{array}{l}\text { Fixed/ } \\
\text { Variable }\end{array}$ & MIDI (range 0 to 1) & Frequency Hz \\
\hline OSC 1 $\mathcal{~ P i t c h ~}$ & Variable & 0 to 1 & $60 \mathrm{~Hz}$ to $4000 \mathrm{~Hz}$ \\
\hline OSC 2 $\mathcal{L}$ Pitch & Variable & 0 to 1 & $60 \mathrm{~Hz}$ to $4000 \mathrm{~Hz}$ \\
\hline Cutoff & Variable & 0.3 to 1 & $60 \mathrm{~Hz}$ to $4000 \mathrm{~Hz}$ \\
\hline Resonance & Fixed & 0 & \\
\hline Brightness & Fixed & 1 & \\
\hline OSC 2 Detune & Fixed & 0.4 & $1600 \mathrm{~Hz}$ \\
\hline OSC 1 Volume & Fixed & 1 & \\
\hline OSC 2 Volume & Fixed & 1 & \\
\hline
\end{tabular}

In the second synthesizer the oscillator used was white noise set to full (1) to create the drum machine clap sound. The cut off filter varied according to the size of the asteroids with smaller collisions having higher Cutoff settings and larger collisions lower Cutoff settings. Table 2 shows the variable and fixed parameters.

Table 2: Sonification design Subtractive Synthesizer 2

\begin{tabular}{|l|l|l|l|}
\hline Parameters & Fixed/ Variable & $\begin{array}{l}\text { MIDI (range 0 to } \\
\text { 1) }\end{array}$ & Frequency Hz \\
\hline Cutoff & Variable & 0.3 to 1 & $60 \mathrm{~Hz}$ to $4000 \mathrm{~Hz}$ \\
\hline Noise volume & Fixed & 1 & \\
\hline Filter amount & Fixed & 0.7 & $2800 \mathrm{~Hz}$ \\
\hline Filter type 24dB & Fixed & - & - \\
\hline Vibrato type & Fixed & 0.6 & $2400 \mathrm{~Hz}$ \\
\hline Brightness & Fixed & 1 & \\
\hline Resonance & Fixed & 0 & \\
\hline
\end{tabular}

Spatialization would be used to give temporal-spatial dimensions and consist of two modes of mapping: One mode for surround sound, the other for stereo. The spatial surround system would be configured to work on a quadraphonic system (four speakers). The sound would have to revolve around the listeners head in the same way that a Kuiper Belt would revolve around a star.

The data consisted of X (horizontal) and Y (vertical) axes. The Y axis would not be used for the testing since the surround sound would only work on a flat horizontal plane. Surround allowed a choice of between 2 to 12 speakers to output the signal. An external soundcard would be needed to be able to connect to more than two speakers. The system allowed the user to choose their own audio 
interface. The sound would move from speaker to speaker by having a crossfader between each channel transmitting from speaker one to four then back to one again and following the same sequence repeatedly during playback. The volume range was set from 0 to 75 as a MIDI value. The range was capped to 75 and not 127 since distortion was occurring above the MIDI value 90. This kept the volume of each channel within a safe limit without peaking.

The stereo panning would be configured to work by shifting from the left to the right speaker in conjunction with a rise in pitch. The sound would move from left $(-1)$ to right $(+1)$ between $60-4000$ $\mathrm{Hz}$. This allowed a listener to hear the differences in intensity of the collisions with larger collisions inclining more towards the left ear and smaller ones more to the right ear. It will also give a more quantifiable impression of the differences in collision.

The synthesizers used in this sound design lacked the required amount of distortion to achieve this goal and sounded flat, lacking the dimension needed to make the cracks sound more realistic. Three effects units were used to achieve this. The first is known as a Glitch Effect, the one used was the Glitchmachines Fracture [35]. This effect mimics a form of distortion created by digital transmission error or a lag in signal causing a signal to stutter and to time-stretch, which is also known as Databending [36]. This effect uses Cutoff filtering and Modulation but also a parameter which is called Stuttering, which creates a jittery type of sound that comes from digital signal corruption. When set at fast speeds the stutter sounds like a Ring Modulation effect which multiplies two waveforms, called carrier and modulator. The resulting signal contains the additions and subtractions of the two signals' frequencies, which are called the upper and lower sideband [37].

The second effect, Glitchmachines Hysteresis [38], adds several different effects which include modulation and further data bending abilities. It also has synthesis aspects such as additional waves and Sample \& Hold which can cause various types of audio signal degradation [39]. On an analogue circuit the capacitor samples the input voltage when the circuit switch is closed. When the circuit switch is open, the capacitor holds that voltage, and this allows other circuits to respond appropriately [40]. This effect added more distortion, and the added waveforms gave further tonal quality to the sound enhancing the timbre and sharpening the signal.

The final effect reverb added a sense of depth to each pulse and smoothed harsh edges of higher frequencies. A Max Reverb Object, gave more impact and the idea of larger sized collisions in the lower pitches, making explosions sound deeper. Two reverbs were routed to work with both the surround and the stereo outputs. The use of reverb in the surround system was routed by having one reverb to spread across both the front and back left channels and the other to spread across the back and front right channels. The tail of one reverb unit would mix into the attack of the second reverb unit due to a long reverb time of 11 milliseconds that was added to both units. The reverb size was set to about a fifth at 19.70 not to be too big and drown the distinction of the pulses but large enough to allow lower pitches to sound larger as they exploded. The added tail gave more of an impression of larger asteroids colliding. The dry to wet ratio was $100 \%$ and helped to give the impression of ice cracking where each break resonates slightly at a certain length of time. When using the stereo system each reverb unit was connected to separate channels to preserve the signal's mono characteristics. The resulting signal would pass through a mono reverb preventing the signal from being outputted in stereo if the left and right channels were connected to the same stereo reverb unit. The interface allowed the user to load the dataset, control playback speed and select data segments for repeat playback. The user controlled the volume from her laptop. A recording device was built into the interface so that $\mathrm{AB}$ could record the sonification and could listen to it on any regular media playback device. The recorded audio files could be saved as WAV, AIFF and FLAC. An example of the sonification can be found online via Soundcloud [41].

\section{Evaluation}

The sonification design was evaluated by $A B$ to test its usability, the efficacy of the parameter mapping and whether any changes were needed. On the day of the evaluation there was a technical problem that hindered the playback. Many attempts were made to try and get the interface working but it kept crashing. Due to this set back the first test was conducted in person with $\mathrm{AB}$ in an office that had been booked for the appointment. The second had to be conducted online about six weeks later after the technical issue had been resolved and a new copy of the interface could be sent to AB. The second test was done as a Skype interview. In both instances the two interviews were recorded on a portable audio recorder.

When the test was conducted in person at a hired office space, a QTX HA-40 four-channel headphone amplifier allowed two pairs of Beyerdynamic DT770 Pro 250 ohms headphones for simultaneous playback. Amplitude was set at a sound pressure level of $65 \mathrm{dBA}$ RMS and $100 \mathrm{dBA}$ peak. Listening levels were $20 \mathrm{~dB}$ below the considered safe level for an eight-hour working day [42].

The evaluation consisted of two parts. The first tested the interface and the second, the efficacy of the sonification design. Each test would last about 90 minutes and were semi-structured. The first test consisted of two sections. The first section consisted of 18 multiple choice questions based on a 5-point Likert scale running from negative (1) to positive (5). These questions focused on the functionality of the interface, it's effectiveness and the validity of each component. The second section consisted of 12 questions about functions that could be added to the interface. The second test consisting of 44 questions enquired about the efficacy and comprehensibility of the sound design and whether any changes to the sonification were required.

The first interview lasted about an hour and a half, 20 minutes of which were spent trying to fix a technical problem. The surround system was receiving too much information at one go when loading the data into the interface. This jammed the system and stalled playback. Several attempts were made to fix the issue, none were successful. The interview was based on an explanation given by the designer. Since the first test was all about the sonification playback interface it was possible to do this without using the interface. The second test was done over Skype and lasted about an hour. The mended interface was sent to $\mathrm{AB}$ along with recordings at various playback speeds to test the sonification before sitting for the final interview. $\mathrm{AB}$ answered all the questions. Both interviews were recorded on a portable audio recorder, transcribed, and coded. 


\section{Results}

In the first evaluation $\mathrm{AB}$ found that the functionality of the interface straightforward: "The interface itself is great! It's a good layout!" She found the interface self-explanatory and easy to use. The loading of the data was taking a while and crashing all the time. $\mathrm{AB}$ classified this as having no opinion about it but put down an extra note on her response sheet suggesting that this function was still bugged. She found the playback to be easy. When asked about the loop function, she had no opinion about this since she wouldn't be using this tool so often. It was easy to change the playback speed and she found it extremely useful that she could switch playback speeds during playback. With regards to the number boxes, she felt that they needed to be labelled according to their unit of measure.

When asked about the color coding of the different functions $\mathrm{AB}$ found it useful, but not color blind friendly. The buttons that were encoded in red and green could be problematic: "You have words, it's not like it wouldn't be useable, but if you are intentionally using green and red to represent different things, they're not color blind friendly". $\mathrm{AB}$ did not find the minimum and maximum collision information useful but did consider the number boxes related to column and rows of data useful for debugging.

The next question asked about the practicality of having a tool that allows users to select certain data segments. $A B$ found this to be extremely useful but stated that it would be more effective if it was labelled according to time instead of line number. She also suggested that if the selection bar had ticks and labels on it then the user would know how to select exact segments of time. AB found the sonification recording function to be especially useful, as it offered flexibility: "You can share with other people without having to go through this. Just put it in your talks and things. Yeah, that's very useful". She also found recording easy to use. AB suggested that if the 3D model was integrated into the sonification interface then it should be able to record the visualization with the sonification. The recording could allow an audience to see and hear activity in relation to that specific point in space and time.

Part two of the first evaluation was aimed to find out whether $\mathrm{AB}$ would like to include additional functionality that was not currently found on the interface (refer to Table 3)

Table 3: Additional functions to be added to interface

\begin{tabular}{|l|l|}
\hline Function & Comments \\
\hline AU & $\begin{array}{l}\text { To use Astronomical units instead of line } \\
\text { numbers }\end{array}$ \\
\hline Parametric EQ & $\begin{array}{l}\text { Allows enhancement or reduction of internal } \\
\text { harmonics to facilitate distinction between small } \\
\text { and large asteroid collisions }\end{array}$ \\
\hline $\begin{array}{l}\text { Additional dataset } \\
\text { channels }\end{array}$ & $\begin{array}{l}\text { Multiple loading systems to load different aspects } \\
\text { of data into interface separately }\end{array}$ \\
\hline Y axis & $\begin{array}{l}\text { Include vertical plane (Y axis) to represent the } \\
\text { effect of a wave created by a planetary trajectory } \\
\text { within the Beta Pictoris Kuiper belt }\end{array}$ \\
\hline Data visualisation & $\begin{array}{l}\text { Include the 3D visualized model of the Beta } \\
\text { Pictoris system and synchronize with the } \\
\text { sonification }\end{array}$ \\
\hline Added functions & $\begin{array}{l}\text { Add rewind, fast forward, pause, scrub and record } \\
\text { (Visualisation) functions }\end{array}$ \\
\hline
\end{tabular}

The second evaluation was conducted sometime after the first. $\mathrm{AB}$ was sent recorded copies of the playback at speeds from 1 to 100 milliseconds. She also received a copy of the interface and the dataset itself so that she could use it to discover different functions. This evaluation was conducted via Skype and lasted roughly about 1 hour. $\mathrm{AB}$ was unable to test the surround sound function of the interface and only got to listen to sonification in stereo.

When first listening to the sonification $\mathrm{AB}$ immediately sought patterns. The sonification was not considered harsh or tiring to listen to. She had expected it to sound like noise but was surprised that the sonification had a structure and was pleasant to listen to. $\mathrm{AB}$ was instantly able to hear differences in pitch and could discern the movement of the planet in the system. She could also hear the directionality of the sound and feel the movement of the collisions taking place at different points in the stereo spectrum.

The next set of questions related to the sonification playback speed. One aspect was that it was hard to tell whether the sonification playback speed had changed with the faster speeds. Up until 10 milliseconds no change was apparent. $\mathrm{AB}$ noticed this and commented about it. By switching between the different playback speeds $\mathrm{AB}$ found that the faster ones gave a general impression of how the collisions were happening. The slower ones allowed her to hear things in more detail. She found that at 100 milliseconds she could get a much broader impression of what was going on. AB felt that the faster speeds would be useful to listen to while doing some other tasks to get the overall impression. At the slower speeds $A B$ was able to notice how the stereo spatial mapping was working and to really feel the differences in the collisions as they manifested around different parts of hearing between left and right ear:

"I would get a burst on one side of my head versus the other, which is really interesting because that implies to me that there are constant collisions happening, that are spatially dependent, which is what I would expect from knowing my data."

$\mathrm{AB}$ noticed there were many more smaller collisions from the higher frequencies and the larger collisions were less frequent but happened in short bursts. The lower frequencies represented high energy collisions and $\mathrm{AB}$ had expected these to be less recurrent. She was he could hear the differences in the low pitch collisions occurring at different locations around her head. Through the sonification she could trace the trajectory of the planet by listening to the lower pitched collisions indicating a larger body passing through the Kuiper belt. AB expected that if that in surround sound the spatial differences would be heard even more clearly.

The next set of questions inquired about the sonic aesthetics. AB considered this well done. She had previous experience in designing collisional sounds and found: "It always just sounds very static to me because I'm picking sounds that are not that nice to listen to." With the sonification design she found: "It does sound like ice breaking but it's also a bit more melodious than just static noise which I appreciated." AB thought the pitch distinction to be especially noticeable despite being like the sound of breaking ice. The sonification helped to picture the collisions of asteroids and the context fitted the dataset. "If it had been some completely different sound, from a different context, it would have been just an extra step for brain to understand what I was listening to." She stated that the aesthetics of the sonification: "had a similar quality to rain 
falling on a roof." This meant that the sonification worked as a natural sound with regards to it not being a static, instead it was continuously modulating. This is an attribute which is inherent in natural sounds, often changing in psychoacoustic qualities: "It did feel more natural rather than artificial. Even though obviously, I knew it was artificial, it didn't sound like anything that I had heard but there was a more natural quality to it."

$\mathrm{AB}$ elaborated further on the relaxing sound of rain falling on the roof and other images that came to mind. She reinforced that she could hear her model and the ice breaking as asteroids collided. She also imagined the sounds of objects falling, rocks clattering down or rain fall, feeling: "A real world parallel of various natural things striking each other in space and things falling to the ground."

The next section queried about the size of the collisions and $\mathrm{AB}$ found that it was easy to hear the differences and distinctions. She could hear loose differences in asteroids that were roughly between one meter all the way to one $\mathrm{km}$. And she could also feel "The realworld picture" of small rocks hitting against each other.

The next question asked whether $\mathrm{AB}$ was able to obtain any new insights from the sonification. Since she had already conducted the study before with the 3D model that she built, she didn't learn anything new from the sonification. She did however state that if she used sonification when she had originally been analyzing her data it would have given her the insight she required. She feels that sonification would have helped her to understand the system a lot better. Initially $\mathrm{AB}$ and her team had struggled a great deal to understand the data until they constructed the 3D model: "And so I think having it (the sonification) as I was analyzing it would have provided me with insights faster. " $\mathrm{AB}$ felt that this would have been the case especially if surround sound was applied.

This led to questions about the spatial representation of the data and its importance for parameter mapping. AB commented that this was most important since the data was spatially dependent. She was especially impressed that even though she could only test the model in stereo she was still able to detect the planet in the Kuiper belt and fully understand the dataset clearly.

Even though the surround sound could not be tested the designer asked $\mathrm{AB}$ if she felt that it would have made a difference to the representation of the model. AB answered:

"I think it would just provide another dimension, another spatial dimension to this. Like left versus right is interesting enough, but you know, adding another dimension spatially to that would, I think, provide even more information as we listen to it."

The data also contained a $\mathrm{Y}$ axis which represented the vertical axis of the Kuiper belt. AB felt that the vertical axis could add another dimension of understanding to the dataset and replicate the planetary movement in more detail. It would be able to show the ripple effect of the vertical peak of the wave of asteroids being tossed about as the planet pushed them away from its trajectory. This led to the question of how surround sound could be used in AB's work. If she was working on similar, multiple projects she: "would consider having speakers outside my office in surround sound". This would only be applicable if she had an office to herself. Astronomers usually move every couple of years until they get a permanent job and often must share an office with co-workers. There was also the disadvantage of having to carry a surround sound system to conferences and public engagements: "And that would be unfortunate because I think that if I heard something that helped me understand the data better, I would want to be able to share it with people." $\mathrm{AB}$ felt that if these practices became more commonplace then conferences would be ready to set up dedicated rooms.

$\mathrm{AB}$ described the spatial data in relation to the collision data. The higher pitched, smaller asteroid collisions were a continuous background sound and the lowest pitches started to come through in bursts varying from the right to the left. It was easier to discern the collisions that were more towards either ear than central:

"I found myself trying to turn my head the way you do when you are trying to locate sound and of course it didn't help but that meant that my brain reacted as if it was a real sound difference."

When asked what $\mathrm{AB}$ liked or disliked about the sonification, and if anything needed changing. $\mathrm{AB}$ enjoyed the spatial aspect: "It made it feel for a moment like I was actually standing in the disc which was great." $\mathrm{AB}$ did not dislike anything about the sonification or have anything further to suggest about the design. She did want to test out the sonification with the $3 \mathrm{D}$ visualization and to control them together. $\mathrm{AB}$ felt that if she was to do all this again, she would provide a smaller version of the dataset of a lower resolution. With the changes suggested to the interface design in the first part of the evaluation she could then use a slider to switch between different time scales as well as watch and listen to what was happening at various moments in time. She also imagined having the visualization to match the logarithmic nature of sound. This would help to achieve a clearer understanding of the radical changes in the Kuiper belt causing a wave to propagate outwards: "And so being able to jump to different times and see the different spatial effects would be interesting in this system." The spatial characteristics of the dataset are an obvious mapping in this dataset:

"If you're standing at a certain radius within the disc and the wave, which you would hear as an increase in those low pitch collisions sweeps by you, that would be interesting if you would be able to hear it. You'd need a different dataset so that you could hear the time."

The idea of being able to switch perspectives between Stereo and Surround would enable the user to hear the wave and its movement from one side to another in the stereo. The surround could be used to hear activity happening in different belt quadrants. Standing at the center in a surround sound system would allow the user to hear the wave recede outwards offering a new angle of perspective.

\section{Discussion}

The sonification accurately represented the data allowing $\mathrm{AB}$ to immediately detect a planet charting through a Kuiper belt. She could clearly hear its path due to explosive collision data. AB could also hear quantifiable differences in the sizes of the collisions and could get a good impression of the sizes of the different colliding bodies. Since the model was only tested in stereo it was surprising to find how immersive $A B$ found the sonification to be. She could picture what was happening in her mind and felt a sense of being in the Kuiper belt and hearing all the activity as the planet passed her [3]. She even mentioned there were times when she wanted to turn her head to see where collisions were happening [4]. The 
sonification did not present anything new but $\mathrm{AB}$ stated that if she had used sonification in the initial development of her 3D model it would have allowed faster detection of the planet and its trajectory.

User centered and task-based design methods were found to be effective for creating a meaningful sonification $[7,8]$. The narrative worked based on mimicking realistic, familiar environmental sounds to create strong associative ties to make it easier to understand what a user is listening to $[10,11,12]$. When querying what data represents it is important to understand how to represent it. The sonification had to mirror data sonically and $\mathrm{AB}$ described it as: "The real-world picture". $\mathrm{AB}$ mentioned that the sonification would complement a 3D visualization she had created. By having such a realistic visualization, one would expect the sound design to act as a realistic or conceptual impression of the asteroid collisions that were taking place within the belt [13]. AB was drawn in by the realism of the sound. By mimicking the ice breaking when asteroids collide, it took $\mathrm{AB}$ less time to decipher what was happening in the dataset. She immediately understood the planet's movement, its mass, and the effect it had on the belt system. The sonification allowed $\mathrm{AB}$ to listen to the data more carefully and to extract meaning from what she was listening to [21, 24]. She paid attention to the ideas, feelings and memories associated to the deep listening that Oliveros [22] describes. This level of engagement and immersion is reflected in AB's comments: "It made it feel for a moment like I was actually standing in the disc which was great."

Realistic impressions were generated by treating objects in the data as dimensional. This means that multiple psychoacoustic mappings were required. As the rock strikes the other rock the impact sound conveys the energy and size of the collision through the timbre of the object. Higher pitched sounds were more brittle and thinner compared to a larger object that would sound less clear and more booming. Sound depends on the space within which it propagates. It is omni-directional and subject to time [6] and in a dataset where asteroids are moving spatial representation is essential.

The techniques used to make a meaningful sonification seemed successful in conveying the data. Human perception and cognition are vague [17] and effective implementation of acoustic representations of data are complex and difficult to understand. However, by studying the significance of an object and its meaningfulness it is possible to create effective sound design [9]. With astronomical data, astrophysicists are looking at new worlds in context. By mimicking these realities, making them reachable and tangible makes data more accessible and relatable. Strengthening associations and immersion allows users to imagine and picture what is going on, possibly leading to new insights.

$\mathrm{AB}$ spoke about public engagement and how she would be interested in sharing a multisensorial representation of the data in audio-visual format. There are limitations that she pointed out with regards to surround sound. Most astronomers share office space and would not be able to use surround sound and to disturb their coworkers. When wanting to share the data at conferences or in public engagement exercises it is not practical to lug a surround sound system around every time and there might be limitations in the presentation space that do not allow such a configuration.
This calls to question how future technologies will shape office or lab environments and further development of multisensorial technologies might reshape the way that astronomers and other scientists carry out their work. It is also worth considering whether the use of surround sound on both the $\mathrm{X}$ and $\mathrm{Y}$ axial planes, should be compromised to suit current hardware accessibility limitations. Due to the technical issues that cropped up, the system was only tested in stereo, and was effective enough to convey the data accurately. But if spatial sound representation is just as effective in other sonification designs, then user might want to consider changing their working environment and allow for suitable spaces or rooms. This whole aspect might even push things on a much broader level where speaker and headset design technology may be revised to suit the new forms of audio replicating use-platforms.

These are all questions to be taken into consideration for creating future sonifications. As multisensorial data platforms become more integrated then sonification will have to suit the purpose. In a data portrayal environment where the user can see, hear, and feel data it could be compared to a video game and this platform of sound design points the way forward for sonification designers to dive into and to even be able to form universal design criteria.

\section{Conclusion}

This sonification was co designed with $\mathrm{AB}$ to represent a portion of the dataset simulation of the $\beta$ Pictoris system across 30,000 days, in an average of two minutes. This allowed $A B$ to quickly run through data and hear it more globally before slowing it down for finer detail. The use of auditory affordances facilitated AB's immediate understanding of the data with no further effort being required. Mimicking qualities of familiar natural sounds allowed $\mathrm{AB}$ to easily understand differences in the sizes of asteroid collisions as she felt that she was listening to the familiar sound of ice breaking.

To establish consistency, the model should be tested with other astronomers in the same field. A surround sound system would have to be tested and the sonification mapped to vertical and horizontal axes. This may give a more accurate representation of the waves forming as the planet knocks asteroids in the belt. Further research into the use of familiar, environmental sounds in sonification design could be explored in the context of other applications. Such as planetary harmonics in self-organising systems, the evolving relationships between planetary systems and even galaxies.

Grounded Theory was effective in this instance for reaping a deeper understanding of AB's relationship to the dataset. The derived keywords and descriptions effectively formed the basis for the sound design, which helped $\mathrm{AB}$ to achieve a clear understanding of the mappings and ensured that the sonification perceptually mirrored the dataset. Within the field of Exosolar planetary research spatialized sonification appears to be a suitable candidate for conveying rich and extensive data. The temporal nature of data is enhanced and put into the context of its spatial dimensions. These characteristics of the sonification design reported in this study seem to be effective when translating astronomical data, which is often temporal-spatial in nature. 


\section{REFERENCES}

[1] Chowning, J. M. (1971). The simulation of moving sound sources. Journal of the audio engineering society, 19(1), 2-6.

[2] Rosli, M. H. W., Cabrera, A., Wright, M., \& Roads, C. (2015). Granular model of multidimensional spatial sonification. In Proceedings of the 12th Sound and Music Computing Conference (SMC-2015).

[3] McLeran, A., Roads, C., Sturm, B. L., \& Shynk, J. J. (2008). Granular sound spatialization using dictionary-based methods. In Proceedings of the 5th Sound and Music Computing Conference, Berlin, Germany (No. 1).

[4] Nasir, T., \& Roberts, J. C. (2007). Sonification of spatial data. Georgia Institute of Technology.

[5] Childs, E., \& Pulkki, V. (2003). Using multi-channel spatialization in sonification: a case study with meteorological data. Georgia Institute of Technology.

[6] Liljedahl, M., \& Fagerlönn, J. (2010, September). Methods for sound design: a review and implications for research and practice. In Proceedings of the 5th audio mostly conference: A conference on interaction with sound (pp. 1-8). dl.acm.org/doi/pdf/2010

[7] Lenzi, S., Terenghi, G., \& Moreno-Fernandez-de Leceta, A. (2020). A design-driven sonification process for supporting expert users in real-time anomaly detection: Towards applied guidelines. EAI Endorsed Transactions on Creative Technologies, 7, 23.

[8] Verona, D. J. (2017). The Effects of Design on Performance for Databased and Task-based Sonification Designs: Evaluation of a Task-based Approach to Sonification Design for Surface Electromyography (Doctoral dissertation).

[9] Hug, D. (2008), Genie in a bottle: Object-sound reconfigurations for interactive commodities, in Proceedings of Audiomostly 2008, 3rd Conference on Interaction with sound,

[10] Blanco, A. L. A., Grautoff, S., \& Hermann, T. (2020). ECG sonification to support the diagnosis and monitoring of myocardial infarction. Journal on Multimodal User Interfaces, 1-12.

[11] Wolf, K., \& Fiebrink, R. (2019). Toward Supporting End-User Design of Soundscape Sonifications.

[12] Vickers, P., Laing, C., Debashi, M., \& Fairfax, T. (2014). Sonification aesthetics and listening for network situational awareness. arXiv preprint arXiv: 1409.5282.

[13] Zettl, H. (2013). Sight, sound, motion: Applied media aesthetics. Cengage Learning. Wadsworth Publishing Company, An International Thomson Publishing Company 1999, Third Edition.

[14] Bødker, S., \& Klokmose, C. N. (2016). Dynamics, multiplicity and conceptual blends in HCI. In Proceedings of the 2016 chi conference on human factors in computing systems (pp. 2538-2548). $\underline{\text { doi/pdf } / 10.1145 / 2858036.2858530}$

[15] Fauconnier, G., \& Turner, M. (2003). Conceptual blending, form and meaning. Recherches en communication, 19(19), 57-86.

[16] MacDonald, D., \& Stockman, T. (2018). Soundtrad, a method and tool for prototyping auditory displays: Can we apply it to an autonomous driving scenario? Georgia Institute of Technology.

[17] Roddy, S., \& Bridges, B. (2020). Mapping for meaning: the embodied sonification listening model and its implications for the mapping problem in sonic information design. Journal on Multimodal User Interfaces, 1-9.

[18] Csikszentmihalyi, M., \& Halton, E. (1981). The meaning of things: Domestic symbols and the self. Cambridge University Press. Chapter 1, $\mathrm{p} 1$. [19] Laurel, B. (2013). Computers as theatre. Addison-Wesley. Second edition, 27 September 2013.

[20] Pauletto, S., Hug, D., Barras, S., \& Luckhurst, M. (2009). Integrating theatrical strategies into sonic interaction design. In 4th Conference on Interaction with Sound: Audio Mostly 2009; Glasgow; United Kingdom; 2 September 2009 through 3 September 2009 (pp. 77-82).

[21] Tuuri, K., \& Eerola, T. (2012). Formulating a revised taxonomy for modes of listening. Journal of New Music Research, 41(2), 137-152.
[22] Oliveros, P. (2005). Deep listening: A composer's sound practice. IUniverse.

[23] Ceraso, S. (2014). (Re) Educating the senses: Multimodal listening, bodily learning, and the composition of sonic experiences. College English, $77(2), 102-123$

[24] Chion, M. (2012). The three listening modes. The sound studies reader, Routledge 2 Park Square, Milton Park, Abingdon, Oxon OX14 4RN 2012, First Edition, p 48-53.

[25] Tuuri, K., Mustonen, M. S., \& Pirhonen, A. (2007). Same sounddifferent meanings: A novel scheme for modes of listening. Proceedings of Audio Mostly, 13-18.

[26] Tie, Y.C., Birks, M., Francis, K. (2019): 'Grounded theory research: A design framework for novice researchers', Sage Open Med v.7 2019, Published online January 2, 2019, doi: 10.1177/2050312118822927

[27] Mills, J., \& Birks, M. (2014). Qualitative methodology: A practical guide. Sage London.

[28] Nesvold, E. R., \& Kuchner, M. J. (2015). A SMACK MODEL OF COLLIDING PLANETESIMALS IN THE $\beta$ PICTORIS DEBRIS DISK. The Astrophysical Journal, 815(1), 61.

[29] Swallow, D. (2012). Live Audio: The Art of Mixing a Show. CRC Press. ISBN 9780240816050, Published September 10, 2012 by Routledge, 256 Pages, Chapter 2 p14.

[30] Einbond, A. (2013). Subtractive Synthesis: noise and digital (un)creativity. In: A. Cassidy \& A. Einbond (Eds.), Noise in and as music. (pp. 57-75). Huddersfield, UK: University of Huddersfield Press. ISBN 9781862181182

[31] Moore, B. C. (2012a). An introduction to the psychology of hearing. Brill. Sixth edition, Koninklijke Brill NV, Leiden, The Netherlands, ISBN 978-90-04-25242-4, p210

[32] Moore, B. C. (2012b). An introduction to the psychology of hearing. Brill. Sixth edition, Koninklijke Brill NV, Leiden, The Netherlands, ISBN 978-90-04-25242-4, p211

[33] Vickers, P. (2013). Ways of listening and modes of being: Electroacoustic auditory display. arXiv preprint arXiv:1311.5880.

[34] Leech, R., \& Saygin, A. P. (2011). Distributed processing and cortical specialization for speech and environmental sounds in human temporal cortex. Brain and language, 116(2), 83-90.

[35] Glitchmachines (n.d.) Fracture, accessed Jan 2021 from: https://glitchmachines.com/products/fracture/

[36] Anderson, A. (2014) 'Creative Sound design: When glitches are a good thing', A Sound Effect, Published $3^{\text {rd }}$ March 2014, Accessed June 2020: asoundeffect.com creative-sound-design

[37] Hoffmann-Burchardi, R. (2008, September). Digital simulation of the diode ring modulator for musical applications. In Proc. Conf. Digit. Audio Effects (pp. 165-168).

[38] Glitchmachines (n.d.), Hysteresis, Accessed Jan 2021 from: https://glitchmachines.com/products/hysteresis/

[39] Pichler, H., \& Skritek, P. (1980). Design Principles of Sample \& Hold Circuits for Digital Audio Systems. In Audio Engineering Society Convention 65. Audio Engineering Society.

[40] Reid, G. (2000), From Sample \& Hold to Sample Rate Converters (1), Sound on Sound Magazine, Published August 2000, accessed June 2020 from: soundonsound.com

[41] Soundcloud, Michael Quinton_Napier: Sonification of asteroid collisions in Beta Pictorius Exosolar System at 5ms playback speed Accessed Jan 2021 from: https://soundcloud.com/michaelquinton napier/sets/sonification-of-asteroid

[42] Moore, B. C. (2012c). An introduction to the psychology of hearing. Brill. Sixth edition, Koninklijke Brill NV, Leiden, The Netherlands, ISBN 978-90-04-25242-4, p156 